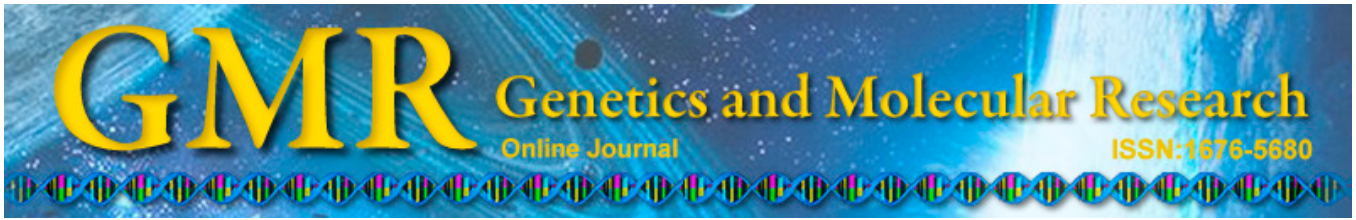

\title{
Developmental changes in the expression of the GLUT2 and GLUT4 genes in the longissimus dorsi muscle of Yorkshire and Tibetan pigs
}

\author{
Y. Liang*, X.M. Yang*, Y.R. Gu, X. Tao, Z.Z. Zhong, J.J. Gong, \\ X.H. Chen and X.B. Lv \\ Sichuan Animal Science Academy, Chengdu, Sichuan, China \\ *These authors contributed equally to this study. \\ Corresponding authors: X.B. Lv / X.H. Chen \\ E-mail: 1vxuebin1965@gmail.com / yzs20070117@126.com
}

Genet. Mol. Res. 14 (1): 1287-1292 (2015)

Received May 24, 2014

Accepted September 25, 2015

Published February 13, 2015

DOI http://dx.doi.org/10.4238/2015.February.13.7

\begin{abstract}
Glucose transporter proteins 2 and 4 (GLUT2 and GLUT4) play important roles in glucose transport and energy metabolism. Changes in the levels of GLUT2 and GLUT4 mRNA were measured in longissimus dorsi muscle from the lean Yorkshire and fat Tibetan pig breeds at six different time points $(1,2,3,4,5$, and 6 months) with quantitative real-time polymerase chain reactions. The results showed that GLUT2 and GLUT4 mRNA were abundantly expressed in the longissimus dorsi muscle and that the developmental expression patterns were similar in both breeds. Tibetan pigs exhibited higher intramuscular fat and GLUT2 mRNA levels, while Yorkshire pigs exhibited a higher myofiber cross-sectional area (CSA) and GLUT4 mRNA levels. Furthermore, the changes in the GLUT4 mRNA levels were strongly and positively correlated with the CSA over a period of six months. These results exhibit time- and breedspecific expression patterns of GLUT2 and GLUT4, which highlight their potential as candidate genes for assessing adipose deposition and
\end{abstract}


muscle development in pigs. These differences in the expression of GLUT family genes may also have indications for meat quality.

Key words: Pig; Developmental expression; Longissimus dorsi muscle; Glucose transporter; Quantitative real-time PCR

\section{INTRODUCTION}

Glucose transport and metabolism are essential for mammalian adipose deposition and myocyte development. However, glucose is unable to permeate the plasma membrane, and its uptake is mediated by a small family of proteins known as glucose transporter proteins (GLUT). These transmembrane proteins equilibrate glucose transport across cell membranes by a passive system of facilitated diffusion (Mueckler, 1990; Smith and Gridley, 1992). To date, at least 14 family members have been identified in mammals, and these have similar structures but different tissue- and cell-specific distributions. GLUT2 and GLUT4 are two of the most important members of the family (Macheda et al., 2005; Montel-Hagen et al., 2008). GLUT2, which has a low affinity for glucose, is mainly expressed in the basolateral membrane of hepatocytes, the kidney, the small intestine, and insulin-producing $\beta$ cells, and it plays a more permissive role in glucose catabolism (Roncero et al., 2004). GLUT4 is the main glucose transporter that is expressed in skeletal muscle and adipose cells, where it plays a key role in cellular glucose uptake following stimulation. For this reason, GLUT4 is referred to as an insulin-sensitive glucose transporter (Gould and Holman, 1993). The results of recent studies (Lee et al., 2002; Kouidhi et al., 2013) have suggested that insulin resistance in glucose transport may be due to defective translocation of GLUT4 in muscle.

Pigs are important animals in the agricultural industry. Porcine muscle has been the focus of research because it represents the most economically important part of the pig carcass. Yorkshire pigs, a typical lean-type Western breed, have been intensively selected for farming over the past two decades for their rapid, large, and efficient accumulation of muscle. In contrast, Tibetan pigs, a typical indigenous Chinese breed living in the Qing-Tibet plateau, and a few other exotic pig breeds have been introduced into the habitat of Chinese native Tibetan pigs because of their geographic isolation. These pigs have low growth rates and less lean meat content but better meat quality than conventional Western pig breeds. Because of their multiple functions in glucose metabolism and muscle development, GLUT2 and GLUT4 may be important candidate genes for improving meat quality. Although the structures and functions of GLUT genes in humans and rats have been widely investigated, there is little information on how changes in their expression could affect adipose deposition and muscle development in pigs. Thus, it is important to investigate the developmental expression patterns of GLUT2 and GLUT4 in the muscle tissues of different pig breeds.

In this study, we investigated the expression patterns of GLUT2 and GLUT4 genes at $1,2,3,4,5$, and 6 months of age in the longissimus dorsi muscle of Yorkshire and Tibetan pigs.

\section{MATERIAL AND METHODS}

\section{Animal and sample collection}

A total of 36 pigs (18 Yorkshire and 18 Tibetan, three each per age group) were used 
in this study. The animals were reared in compliance with national regulations for the human care and use of animals in research. The animals were allowed access to feed and water ad libitum under normal conditions, and they were humanely sacrificed, as necessary, to minimize suffering. The longissimus dorsi muscle near the third or fourth last ribs was carefully and manually dissected from each of the 36 cleaved pigs. All samples were immediately frozen in liquid nitrogen and stored at $-80^{\circ} \mathrm{C}$ until analysis.

\section{RNA extraction and reverse transcription}

Total RNA was extracted with the mirVana Kit (Life Technologies Corporation, Grand Island, NY, USA) from the 36 tissue samples following the manufacturer protocol. The RNA was reverse transcribed into cDNA with the oligo (dT) primer and random primer provided in the reverse transcription (RT) Kit (TaKaRa Bio, Inc., Otsu, Japan) for RT-polymerase chain reaction (PCR) according to the recommendations of the manufacturer.

\section{Myofiber cross-sectional area and intramuscular fat content measurements}

The longissimus dorsi muscle samples were sliced at a thickness of $8 \mu \mathrm{m}$ with a KD2508 rotary microtome (Kedee Instruments Co., Ltd., Zhejiang, China) according to the frozen section procedure described by Wilson (1905) and stained with hematoxylin and eosin (H\&E). The cross-sectional areas (CSAs) were determined for an average of 100 fibers in randomly selected fields with a BK5000 fluorescent microscope (Optec Technology, Ltd., Hong Kong, China) and the Motic Images Advanced 3.2 software. The intramuscular fat (IMF) content was measured by the Soxhelt extraction method with the Buchi extraction system (Büchi Labortechnik AG, Flawil, Switzerland).

\section{Q-PCR assays}

Q-PCR was performed with the SYBR Green PCR kit (TaKaRa Bio, Inc.) on a CFX96 Real-Time PCR Detection System (Bio-Rad Laboratories, Inc., Hercules, CA, USA). Each reaction comprised $12.5 \mu \mathrm{L}$ of SYBR Green q-PCR Super Mix, $2 \mu \mathrm{L}$ of cDNA, $10 \mathrm{pM}$ of each primer, and RNA-free water (Life Technologies Corporation) to a total volume of $25 \mu \mathrm{L}$. The real-time PCR program started with a 30 -s denaturation at $95^{\circ} \mathrm{C}$, which was followed by 40 cycles of $5 \mathrm{~s}$ of denaturation at $95^{\circ} \mathrm{C}$, and $10 \mathrm{~s}$ of annealing/elongation at the annealing temperature (Tm) for each specific primer (Table 1), during which fluorescence was measured. The specific PCR products were confirmed by a melting curve analysis; this allowed for the verification of the presence of one gene-specific peak and the absence of primer dimers. All measurements contained a negative control (no cDNA template), and each RNA sample was analyzed in triplicate. Each objective gene was normalized to $A C T B, T B P$, and $T O P 2 B$. The normalized factors of internal control genes and relative quantities of objective genes were analyzed with the geNorm software (Vandesompele et al., 2002). Data were analyzed by oneway ANOVA and are reported as means $\pm \mathrm{SD}$ for $\mathrm{N}=3$ animals per group. All statistical analyses were performed with the SAS 9.1. 
Table 1. Primers used for quantitative polymerase chain reaction.

\begin{tabular}{|c|c|c|c|}
\hline Gene symbol & Sequences of primers $\left(5^{\prime} \rightarrow 3^{\prime}\right)$ & Temperature $\left({ }^{\circ} \mathrm{C}\right)$ & GeneBank accession No. \\
\hline$\overline{\text { GLUT2 }}$ & $\begin{array}{l}\text { F: GTTCATGGTGGCCGAGTT } \\
\text { R: ATTGCGGGTCCAGTTGC }\end{array}$ & 58 & NM_001097417.1 \\
\hline GLUT4 & $\begin{array}{l}\text { F: GACTCTGGGTGAAAGGG } \\
\text { R: GGGAAGGCTGAGTGAGA }\end{array}$ & 56 & NM_001128433.1 \\
\hline$A C T B^{*}$ & $\begin{array}{l}\text { F: TCTGGCACCACACCTTCT } \\
\text { R: TGATCTGGGTCATCTTCTCAC }\end{array}$ & 60 & DQ178122 \\
\hline$T B P^{*}$ & $\begin{array}{l}\text { F: GATGGACGTTCGGTTTAGG } \\
\text { R: AGCAGCACAGTACGAGCAA }\end{array}$ & 60 & DQ178129 \\
\hline TOP2B* & $\begin{array}{l}\text { F: AACTGGATGATGCTAATGATGCT } \\
\text { R: TGGAAAAACTCCGTATCTGTCTC }\end{array}$ & 60 & AF222921 \\
\hline
\end{tabular}

\section{RESULTS AND DISCUSSION}

When comparing breeds, Yorkshire pigs had a higher CSA than Tibetan pigs from 1 to 6 months. However, the IMF content of Tibetan pigs was higher than that of Yorkshire pigs (Figure 1). These results correspond to their breeding characteristics and distinct breeding history. In Yorkshire pigs, the growth and development of skeletal muscle is greater than that of Tibetan pigs, whereas Tibetan pigs have higher adipose deposition.
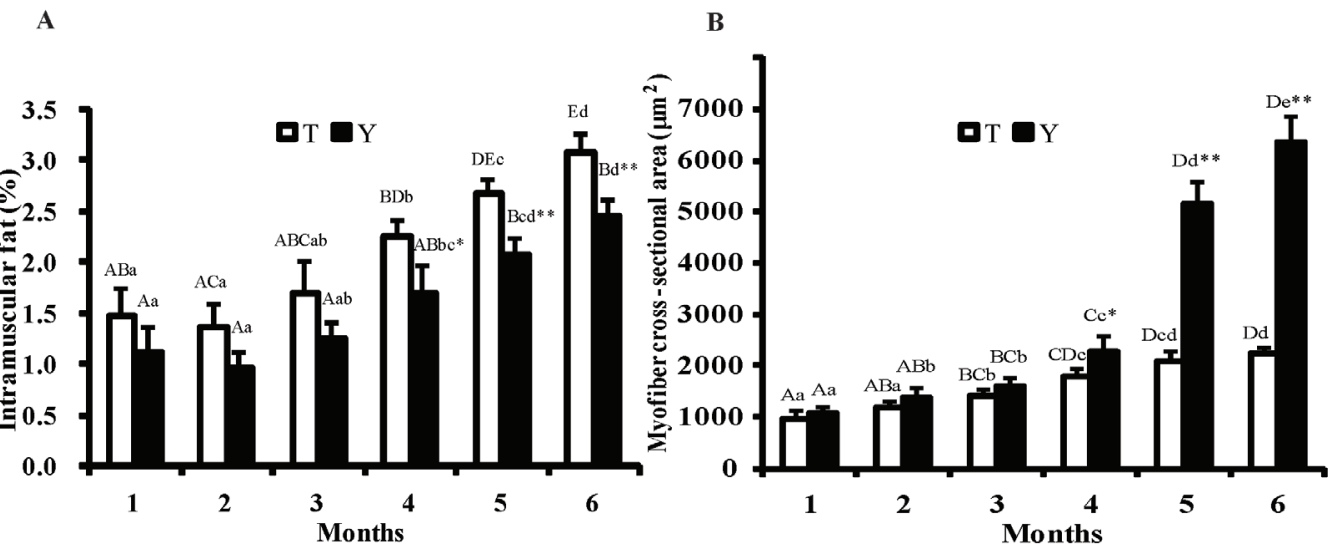

Figure 1. Intramuscular fat (IMF) and cross-sectional area (CSA) differences between breeds and months. (A) Changes in IMF in Tibetan and Yorkshire pigs; (B) Changes in the CSA of both pig breeds. *or **indicates $5 \%$ or $1 \%$ significant differences, respectively, between both breeds at the same age; a different letter indicates a significant difference at the $5 \%$ (small letter) or 1\% (capital letter) levels between months of the same breed. Breed: $\mathrm{Y}$ and $\mathrm{T}$ represent the Yorkshire and Tibetan pigs, respectively.

The developmental expression changes of GLUT2 and GLUT4 genes were investigated by RT-PCR in the longissimus dorsi muscle of both pig breeds from 1 to 6 months of age. In this period, our study showed that the GLUT2 and GLUT4 mRNAs were abundantly expressed in longissimus dorsi muscle and that the developmental expression patterns were similar in both breeds. The developmental changes in GLUT2 expression gradually decreased at first and then increased rapidly in both breeds. The reversal points were at 4 months and 5 months of age in the Tibetan pigs and Yorkshire pigs, respectively. These results corresponded to the regular patterns of their growth and development. Before Tibetan pigs reach 4 months 
of age, their muscle development is more predominant than adipose deposition, with the adipose deposition becoming stronger after 4 months of age. For Yorkshire pigs, this time point comes at 5 months of age. The general trend in the GLUT4 mRNA expression levels from 1 to 6 months increased gradually in the two pig breeds, whereas the expression levels dropped slightly at 2 months of age, probably due to weaning stress. This result was consistent with the findings of Zuo et al. (2010), who have reported that porcine skeletal muscle maintains a high level of expression of GLUT4 mRNA after weaning and that this might have important functions during adult development.

Furthermore, we found that the GLUT2 mRNA expression levels were higher in the fatty Tibetan pigs than in the leaner Yorkshire pigs from 1 to 6 months and that the expression levels were significantly different at 6 months of age $(P=0.033)$ (Figure 2). This was consistent with the biological function of GLUT2, which promotes the differentiation of adipocytes (Kaestner et al., 1989). Accordingly, the IMF of Tibetan pigs was higher than that of the Yorkshire pigs. These results suggested that GLUT2 could regulate lipid metabolism. However, GLUT4 exhibited higher mRNA levels in the leaner Yorkshire pigs compared to the fatty Tibetan pigs from 1 to 6 months, and the expression levels showed significant differences at 6 months of age $\left(p_{6 \text { month }}=6.68 \times 10^{-3}\right)$. The higher GLUT4 mRNA levels in Yorkshire pigs suggested that Yorkshire pigs may need more glucose as an energy source for the development of muscle fibers because GLUT4 is the main glucose transporter and it plays a crucial role in cellular glucose uptake and the regulation of glucose homeostasis in the skeletal muscle of mammals (Shepherd and Kahn, 1999; Kono et al., 2005). Previous studies have demonstrated that increased levels of GLUT4 facilitates glycogen synthesis and is associated with enhanced muscle glucose transport and glycogen storage (Kraniou et al., 2000). Accordingly, the CSA was lower in Tibetan pigs than in the Yorkshire pigs at the same ages. Moreover, the expression changes in GLUT4 $\left(r=0.95, \mathrm{P}=2.34 \times 10^{-6}\right)$ showed a strong positive correlation with CSA. This was consistent with previous findings that the GLUT4 is closely associated with muscle fiber growth and development (Gaster et al., 2000). These results suggested that GLUT4 has an important role in porcine muscle fiber development. Surprisingly, we found that GLUT2, which was usually regarded as having no expression in muscle, was expressed in porcine longissimus dorsi muscle. The possible reasons for this were that the IMF contents of the two researched pig breeds were abundant in the muscle and that qRT-PCR was more sensitive. Further customized surveys should also be performed.

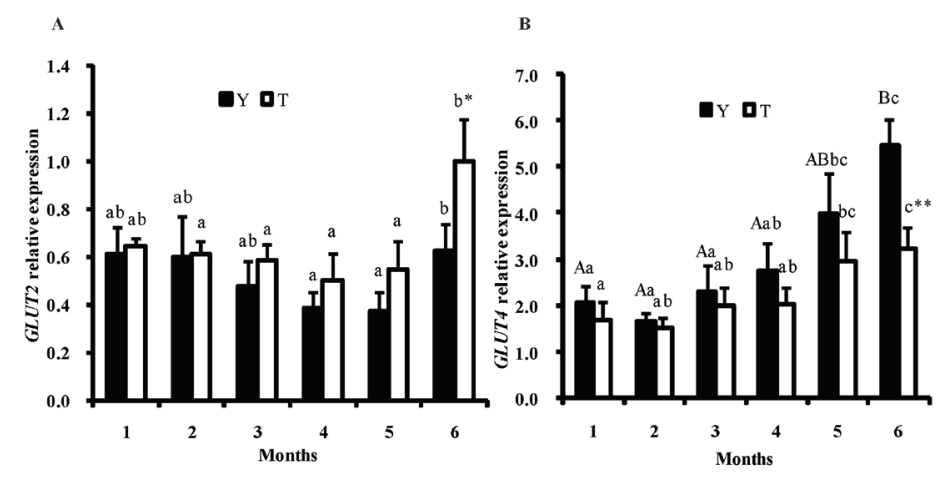

Figure 2. Breed- and month-specific gene expression patterns of (A) GLUT2 and (B) GLUT4. For details, see Figure 1. 
In summary, our findings suggested that GLUT2 and GLUT4 are important for porcine adipose deposition and muscle development and could be regarded as promising candidate genes that are involved in pig growth and meat quality. This study also provides data for further study on the mutual regulation mechanism of genes in the GLUT family.

\section{ACKNOWLEDGMENTS}

Research supported by the Fund of Sichuan Provincial Basic Scientific Research (\#SASA2013A03), the Key Technology Research and Development Program of Sichuan (\#2011NZ0099-2), and the Key Projects in the National Science \& Technology Pillar Program (\#2011BAD28B01).

\section{REFERENCES}

Gaster M, Handberg A, Beck-Nielsen H and Schroder HD (2000). Glucose transporter expression in human skeletal muscle fibers. Am. J. Physiol. Endocrinol. Metab. 279: E529-538.

Gould GW and Holman GD (1993). The glucose transporter family: structure, function and tissue-specific expression. Biochem. J. 295: 329-341.

Kaestner KH, Christy RJ, McLenithan JC, Braiterman LT, et al. (1989). Sequence, tissue distribution, and differential expression of mRNA for a putative insulin-responsive glucose transporter in mouse 3T3-L1 adipocytes. Proc. Natl. Acad. Sci. U. S. A. 86: 3150-3154.

Kono T, Nishida M, Nishiki Y, Seki Y, et al. (2005). Characterisation of glucose transporter (GLUT) gene expression in broiler chickens. Br. Poult. Sci. 46: 510-515.

Kouidhi S, Berrhouma R, Rouissi K, Jarboui S, et al. (2013). Human subcutaneous adipose tissue Glut 4 mRNA expression in obesity and type 2 diabetes. Acta. Diabetol. 50: 227-232.

Kraniou Y, Cameron-Smith D, Misso M, Collier G, et al. (2000). Effects of exercise on GLUT-4 and glycogenin gene expression in human skeletal muscle. J. Appl. Physiol. 88: 794-796.

Lee JS, Bruce CR, Tunstall RJ, Cameron-Smith D, et al. (2002). Interaction of exercise and diet on GLUT-4 protein and gene expression in Type I and II rat skeletal muscle. Acta Physiol. Scand. 175: 37-44.

Macheda ML, Rogers S and Best JD (2005). Molecular and cellular regulation of glucose transporter (GLUT) proteins in cancer. J. Cell. Physiol. 202: 654-662.

Montel-Hagen A, Blanc L, Boyer-Clavel M, Jacquet C, et al. (2008). The Glut1 and Glut4 glucose transporters are differentially expressed during perinatal and postnatal erythropoiesis. Blood 112: 4729-4738.

Mueckler M (1990). Family of glucose-transporter genes: implications for glucose homeostasis and diabetes. Diabetes 39: 6-11.

Roncero I, Alvarez E, Chowen JA, Sanz C, et al. (2004). Expression of glucose transporter isoform GLUT-2 and glucokinase genes in human brain. J. Neurochem. 88: 1203-1210.

Shepherd PR and Kahn BB (1999). Glucose transporters and insulin action-implications for insulin resistance and diabetes mellitus. N. Engl. J. Med. 341: 248-257.

Smith DE and Gridley T (1992). Differential screening of a PCR-generated mouse embryo cDNA library: glucose transporters are differentially expressed in early postimplantation mouse embryos. Development 116: 555-561.

Vandesompele J, De Preter K, Pattyn F, Poppe B, et al. (2002). Accurate normalization of real-time quantitative RT-PCR data by geometric averaging of multiple internal control genes. Genome Biol. 3: Research0034.

Wilson, LB (1905). A method for the rapid preparation of fresh tissues for the microscope. J. Am. Med. Assoc. $45: 1737$.

Zuo JJ, Dai FW, Feng DY, Cao QY, et al. (2010). Molecular cloning and mRNA expression of the porcine insulinresponsive glucose transporter (GLUT4). Asian-Aust. J. Anim. Sci. 23: 640-648. 